\title{
Development Strategies for Creating a Suite of Reference Materials for the In-Situ Microanalysis of Non-conventional Raw Materials
}

\author{
A.D. Renno, ${ }^{*}$ S. Merchel, ${ }^{* *}$ P.P.Michalak, ${ }^{*}$ F. Munnik**, and M. Wiedenbeck*** \\ * Institute of Mineralogy, TU Bergakademie Freiberg, D-09596 Freiberg, Brennhausgasse 14, \\ Germany \\ ** Institute of Ion Beam Physics and Materials Research, Helmholtz-Zentrum Dresden-Rossendorf \\ e.V., D- 01314 Dresden, Germany \\ *** GFZ German Research Centre for Geosciences, Helmholtz Centre D-14473 Potsdam, Potsdam, \\ Germany
}

The availability of reference materials (RM) for microanalytical methods is decreasing. According to [1] currently exist no materials for which the chemical composition has been certified at the $\mu \mathrm{m}$ sampling scale. Many labs use in-house „standards“ as a temporary measure.

Recent economic trends regarding the supply of rare metals readily justify scientific research into non-conventional raw materials, where a particular need is a better understanding of the relationship between mineralogy, microstructure and the distribution of key metals within ore deposits (geometallurgy). Non-conventional raw materials include everything different from what most people consider to be usual or normal, the geometallurgy concept aims to close the gap between ore body exploration/ exploitation (mining) on the one hand, and mineral processing and metallurgy on the other hand. This interface is widely regarded as an interdisciplinary divide that holds significant potential to utilize non-renewable mineral resources more efficiently and sustainably.

Achieving these goals will require an extensive usage of in-situ microanalytical techniques capable of spatially resolving material heterogeneities which can be key for understanding better resource utilization. The availability of certified reference materials (CRMs) is an essential prerequisite for

(1) validating new analytical methods,

(2) demonstrating data quality to the contracting authorities,

(3) supporting method development and instrument calibration, and

(4) establishing traceability between new analytical approaches and existing data sets.

This need has led to the granting of funding by the European Union and the German Free State of Saxony for a program to develop such reference materials .

This effort will apply the following strategies during the selection of the future CRMs:

(1) We will use exclusively synthetic minerals, thereby providing large volumes of homogeneous starting material, following the idea "one CRM for all methods".

(2) We will focus on matrices which are capable of incorporating many 'important' elements while avoid exotic compositions which would not be optimal matrix matches.

(3) We will prefer those phases which remain stable during the various microanalytical procedures.

We decided to synthesize a Fe-S phase, a mineral of the feldspar group, and a phase of the columbite-tantalite solid solution series.

As far as possible, both definitive methods as well as methods involving matrix corrections will be used for determining the compositions of the individual materials. 
The following methods are considered in our planned studies thus far:

- Electron microprobe analysis (EPMA)

- Laser ablation - ICP-MS (LA-ICP-MS)

- Secondary Ion Mass Spectrometry (SIMS)

- Micro-XRF ( $\mu$-XRF)

- Synchrotron-based XRF (S-XRF)

- Particle-induced X-Ray Emission analysis (PIXE)

- Particle-induced $\gamma$-Ray-Emission (PIGE)

- Rutherford Backscattering Spectrometry (RBS)

- Elastic Recoil Detection Analysis (ERDA)

- Nuclear Reaction Analysis (NRA)

A critical challenge for the certification process will be the validation of the determination of analytes concentrations as sub- $\mu \mathrm{g}$ sampling masses.

This initiative will assess the homogeneity of the reference materials at sampling sizes ranging between 50 and $1 \mu \mathrm{m}$. It is intended to document crystal structural and crystal chemical homogeneity too, knowing that these features may potentially impact specific analytical methods, by affecting diffusion, ion conductivity, and subsurface charging. Both spectroscopic and diffractometric methods will be used, like Raman microspectroscopy, FT-IR microspectroscopy, cathodoluminescence spectroscopy, as well as Electron backscatter diffraction (EBSD).

It is planned to cooperate with those who are interested in the development of such reference materials and we invite them to take part in round-robin exercises [2].

\section{References}

[1] http://www.nano-refmat.bam.de/en/: „Nanoscaled Reference Materials“

[2] This project is supported by the European Union (ESF) and the Free State of Saxony. We owe special thanks to all of our colleagues maintaining and running the equipment we used and will use during these project. Many thanks to the custodians of the Geoscientific Collections in Freiberg for saving and providing an invaluable source of material. Motivating discussions on several facets of this study with Jens Gutzmer, Jens Götze, Lutz Hecht, Dieter Rhede, Thomas Wenzel, Jürgen Börder, Thomas Schleid, and Dietrich Heger were very helpful. 